\title{
LETTERS
}

\section{Is suicidality associated with acetylcholine?}

Mukadam et al. (2008) report cholinesterase inhibitors to be effective in treating delirium. This suggests that cholinesterase inhibitors have a broader spectrum of effect than the treatment of dementia alone. Acetylcholine itself interacts with various other neurotransmitters. Cancelli et al. (2004) report that a fall in acetylcholine levels, as occurs in dementia, could increase sensitivity to serotonin agonists. Increased sensitivity to serotonin agonists and cholinergic hypoactivity seems to trigger complex visual hallucinations. It has been reported that selective serotonin reuptake inhibitors (SSRIs) may provoke suicidality, especially in young patients. SSRIs may decrease sensitivity of serotonin agonists and raise acetylcholine levels.

For that reason we hypothesize that suicidality may be associated with augmented acetylcholine levels. Cholinesterase inhibitors augment acetylcholine levels and may thereby raise the risk of suicidality. This corresponds to the observation that these medications may induce psychosis (Cancelli et al., 2004). With respect to glutamate, there is evidence that diminished glutamate levels may induce psychoses. Administration of glutamate antagonists may increase the risk of psychosis. For that reason, non-use of glutamate antagonists and cholinesterase inhibitors seems to be appropriate in the treatment of suicidal or psychotic patients.

\section{References}

Cancelli, T., Marcop, G. and Balestrieri, M. (2004). Factors associated with complex visual hallzcinations during antidepressant treatment. Human Psychopharmacology, 19, 577-584.

Mukadam, N., Ritchie, C. W. and Sampson, E. L. (2008). Cholinesterase inhibitors for delirium: what is the evidence? International Psychogeriatrics, 20, 209-218.

\section{Helmut Niederhofer}

Child and Adolescent Psychiatry, Regional Hospital of Bolzano, Italy

Email: helmutniederhofer@yahoo.de

\section{Confirmatory factor analysis of the Revised Scale for Caregiving Self-Efficacy in a sample of dementia caregivers}

The number of studies aimed at analyzing caregiving distress among carers of people with dementia has grown exponentially in recent years. The study of 